\title{
Leaving the third dimension: no measurable evidence for cognitive aftereffects of stereoscopic 3D movies
}

\section{Q1 Klaas Bombeke Jan Van Looy Arnaud Szmalec Wouter Duyck}

\begin{abstract}
Stereoscopic 3D (S-3D) is becoming an increasingly important display technology. Parallel to this, concern about the potential negative effects of exposure to S-3D movies has been growing. Some manufacturers place disclaimers on their TVs advising people to limit the time they watch S-3D. However, surprisingly little experimental research has been conducted estimating the genuineness of these concerns. Therefore, an experiment was designed to assess the potential impact of viewing an S-3D movie on visual, spatial, and general attention performance. To mimic the real-world experience of watching a movie in the living room, participants $(N=61)$ watched a full movie in either 2D or S-3D. Our results do not show evidence for cognitive aftereffects of S-3D movies. A second experiment $(N=32)$ that focused on possible aftereffects on visual attention also failed to find reliable effects. We therefore conclude that cognitive functioning is not altered by watching an S-3D movie, at least not to an extent that is measurable through well-established cognitive tasks.
\end{abstract}

Keywords - stereoscopic 3D, aftereffects, spatial cognition, visual cognition, mental rotation, change detection, visually directed walking task.

DOI \# 10.1002/jsid.164

\section{Introduction}

Year after year, more S-3D movies and computer games are released and many TV manufacturers are building televisions that are able to display S-3D images. Hence, the question arises if this technological evolution presents health hazards to our cognitive (i.e., information processing) system. Although major companies place warnings in their S-3D television manuals, ${ }^{1}$ hitherto little scientific research has been conducted into its actual aftereffects. As it is conceivable that the near future, people will watch television in S-3D for many hours, it is necessary to know whether cognitive aftereffects emerge after a certain period of time. In this respect, the domain of spatial cognition is particularly relevant. Many people will engage in visuospatial activities such as driving a car after watching a stereoscopic movie. In this study, we therefore assess if aftereffects of stereoscopic 3D can be found using validated, well-known cognitive tests without compromising ecological validity or the extent to which the experimental conditions approximate the real-world experience. ${ }^{2}$

The principle behind S-3D is to present different images to each eye to mimic the binocular disparity that is inherent to the human visual system (e.g., $\left.{ }^{3}\right)$. Binocular disparity creates depth perception, because the brain integrates two slightly different images into a single $3 \mathrm{D}$ percept. ${ }^{4}$ There are many studies dealing with visual discomfort due to S-3D viewing. ${ }^{5-7}$ Emoto, Nojiri, and Okano ${ }^{8}$ asked participants to watch a stereoscopic movie with polarized glasses for $60 \mathrm{~min}$. Afterwards, they assessed subjective visual fatigue and changes in fusional amplitude. Fusional amplitude is a measure that indicates how good the eyes can fuse separate monocular images into one binocular image. The authors found the fusional amplitude decreased after S-3D, indicating a temporary adaptation to S-3D. After a short relaxation period, the fusional amplitude recovered to a pretesting level. Inoue and $\mathrm{Ohzu}^{9}$ found that the time to accommodate (i.e., focus) to a real world object differed when a person had just looked at S-3D images. In contrary, Yano, Ide, Mitsuhashi, and Thwaites ${ }^{6}$ only occasionally found changes in accommodation after 60 min of watching stereoscopic images. In sum, nearly all of these studies have been conducted in the field of vision research by using optometric measurements. These can be considered as the reflection of low-level perceptual processes, that is, the physiological changes on the level of the eyes. When light has reached the retina, the optic nerve sends a signal to the visual cortex in the brain. ${ }^{10}$ However, to recognize the visual field, the images also need to be interpreted. This interpretation process is an example of a cognitive process. In short, cognitive measurements can be seen as reflections of higher-level, postperceptual processes.

Given that perception is a prerequisite for subsequent cognitive processing, it could be expected that visual (fatigue) effects (on the perceptual level) also affect cognitive processing performance. Moreover, we know from research into virtual environments (such as gaming research) that "reentry problems" may occur. ${ }^{11,12}$ Reentry problems refer to perceptual, cognitive,

Received 05/11/12; accepted 05/05/13.

Klaas Bombeke, Research Group Media \& ICT, Department Communication Studies, Korte Meer 7, 9000 Gent, Belgium; e-mail: Klaas.bombeke@ugent.be. K. Bombeke and J. V. Looy are with the iMinds-MICT-UGent, Ghent University, Ghent, Belgium.

A. Szmalec is with the Psychological Sciences Research Institute, University of Louvain, Louvain-la-Neuve, Belgium.

W. Duyck is with the Department of Experimental Psychology, Ghent University, Ghent, Belgium.

(C) Copyright 2013 Society for Information Display 1071-0922/13/0164\$1.00. 
and social disturbances when a person leaves the virtual environment and reenters the real world.$^{13}$ We assume that reentry problems are conceptually similar to aftereffects because both arise when adapting to situations with different perceptual and cognitive demands.

This is, to the best of our knowledge, the first empirical attempt at investigating cognitive aftereffects of S-3D. Hence, in the absence of an established theoretical approach, we conducted an experiment by using multiple cognitive performance measures. The first measurement was the performance in mental rotation or the ability to look at an object from another side without physically turning it first. Shepard and Metzler ${ }^{14}$ discovered that the time to judge if an object is equal to an identical reference object increases in an almost linear way with the amount of rotation of that object. Their theory was that participants turn the figure in their head at a fixed tempo: the larger the rotation, the longer it will last. We chose mental rotation because it is considered a complex form of spatial cognition ${ }^{15}$ and because it has certain generalizability. For example, Stransky, Wilcox, and Dubrowski ${ }^{16}$ found that mental rotation training generalized to surgical tasks indicating that any mental rotation deterioration due to stereoscopic viewing can have real-life consequences. Although the mental rotation task is a well-established measure of spatial cognition, we also wanted to use a more ecologically valid measure of spatial cognitive abilities. Therefore, we also adopted the "visually directed walking task". ${ }^{17-19}$ In this task, people have to estimate distances ranging from 1 to $20 \mathrm{~m}$. It relies on the ability to mentally estimate spatial distances and is an important skill when driving a car for example. Our third function of interest, nonspatial visual cognition was measured with a variant of the visual binding task, ${ }^{20}$ which will be called the change detection task in the following paragraphs. This task is considered as a purely visual task and is mostly used in research into visual working memory. ${ }^{20}$ We implemented this task to check if S-3D has an effect on visual cognition besides spatial cognition. In this task, participants see a configuration of a number of colored squares for a short time and have to indicate if a subsequent configuration of squares is the same or not. Finally, the fourth task in our experiment was unrelated to spatial or visual cognition and was implemented to assess the selectivity of possible aftereffects. An effect of S-3D on this task, would be an indication of general fatigue caused by S-3D. We chose a classic initial letter verbal fluency task whereby the participant had to name as many words as possible starting with a certain letter in $1 \mathrm{~min}$. Research has indicated that verbal fluency is a sensitive measure of overall cognitive fatigue. ${ }^{4}$

To investigate the possible influence of S-3D on these cognitive processes, we chose a mixed factorial design. To control for learning and priming effects, we used a Solomon four-group design. ${ }^{21}$ This design consists of four different conditions. The first two conditions are the same as in classic pretest-posttest designs: participants do a pretest and a posttest, and in between the variables of interest are manipulated. The last two conditions differ in that there is no pretest. By using this design, we could control for possible pretest sensitization effects. ${ }^{22}$ In our study, the independent variable was movie version. Participants saw one out of four movies either in 2D or in S-3D. We chose movies with a realistic playtime, to emulate real-life viewing circumstances. We tested the aftereffect of active S-3D technology whereby the display alternately sends a dedicated image to each eye by shutting down the other eye by using shutter glasses that are synchronized with the screen.

\section{Experiment 1}

\subsection{Method \\ 2.1.1 Participants}

Interested candidates filled out an online survey in which they were asked for gender, age, handedness, gaming experience, optical deviations, and experience with S-3D movies. By doing this, we were able to select 64 participants who were matched on S-3D movie and gaming experience. People with optical deviations (no or very poor vision in one eye, strabismus, presbyopia, etc.) were excluded from participation. The gender ratio was equally distributed over conditions. The participants were on average 19.93 years old $(S D=4.31)$ and were paid $€ 8$.

\subsubsection{Experimental set-up}

Images were displayed on Philips 46" 9000 LED series televisions with a diagonal size of $117 \mathrm{~cm}$ and a resolution of $1920 \times 1080$ pixels. The set-up included two wireless synchronized active LED shutter glasses. The viewing distance was $2.50 \mathrm{~m}$ and the television was placed on a platform, $1 \mathrm{~m}$ above the ground. We used movies that were available both in 2D and S-3D: "Legend of the guardians" (97 min), Alice in Wonderland (102 min), "Sanctum" (107 min), and "Step-up 3" (105 min). Our aim thereby was to use a representative set of the spectrum of available movies in S-3D and avoid materialspecific effects that prevent generalization. Each participant was tested on four tasks. Task version was a within-subject factor and was counterbalanced over participants.

\subsubsection{Mental rotation task}

This task was based on the Vandenberg and Kuse ${ }^{23}$ mental rotation test in which geometrical figures were formed by 10 identical cubicles. In this experiment, we showed 80 different pairs of figures per test session. The second figure in a pair could be either a rotated version or a completely different version of the first figure. A trial started with a fixation cross for about $500 \mathrm{~ms}$, followed by the stimulus pair. The participant had to press as fast as possible a left or right button to indicate if the two figures were the same or not. Half of the trials were correct, half of them were incorrect. Feedback was given when the answer was wrong ("Wrong!"). The dependent variable was the reaction time. 


\subsubsection{Distance estimation task}

The second task was the "visually directed walking task". ${ }^{17}$ A subject stood at a starting point in the hallway of the university building. The experimenter showed a target position at a distance between 1 and $10 \mathrm{~m}$ by placing a cardboard tube on the ground. The subject had to look carefully for $3 \mathrm{~s}$ and afterwards he was blindfolded. Next, he had to walk as close as possible to the target position. The dependent variable in this experiment was the final deviation between the end position of the participant and the defined target position. The distances we used in our research were $2.25,3.60,5.50,7.25,8.10$, and $9.30 \mathrm{~m}$ resulting in six trials per test session. The order of distances was randomized for each participant.

\subsubsection{Change detection task}

In our variant of the Luck and Vogel task, ${ }^{20}$ subjects saw either $2,3,4,6,8$, or 12 colored squares on the screen for $100 \mathrm{~ms}$ (the sample array). The colors were pseudorandomly chosen out of seven colors (pseudo in the sense that the same colors were used per number of squares in the pretest and posttest). After a blank interval of $900 \mathrm{~ms}$, a test array appeared and the participant had to decide as fast as possible

F1 if the stimuli were the same as in the sample array (Fig. 1). In $50 \%$ of the trials, the test array was the same as the sample array. In the other $50 \%$, the test array differed in the color of one of the squares. There were two replications of 48 randomized different trials, resulting in 96 trials per test session.

\subsubsection{Verbal fluency task}

To control for general cognitive fatigue, we implemented a classic verbal fluency task. ${ }^{24}$ We asked subjects to generate as many words as possible in $60 \mathrm{~s}$. There were four trials of 60 s with the letters "E","I", "W", and "L". Every participant did two trials in the pretest and two trials in the posttest, and letter order was counterbalanced over subjects. The dependent variable was the total number of correct responses.

\subsubsection{Procedure}

At their arrival, participants filled out an informed consent. Participants in condition 1 and 2 started with the pretest tasks of which the order was counterbalanced. Afterwards, participants went to a separate room and watched the movie side-by-side in a comfortable chair. Only two people could watch a movie at the same time to avoid effects of seating position. ${ }^{25}$ They were also instructed not to talk to each other during the entire playtime. The room was completely dark, so there was no disturbing influence of sunlight or direct current sources (e.g., fluorescent tubes) on the shutter mechanisms of the active S-3D system. People were strictly forbidden to take off their glasses. The experimenter frequently inspected the setting to make sure that the participants complied with the respected rules. After the movie, participants from all four conditions went through the posttests.

\subsection{Results}

\subsubsection{Mental rotation task}

The first ten trials of every participant were removed because they were considered as the training phase. Next, we removed all trials with an incorrect response. We then checked if our reaction times (RTs) were normally distributed. We saw that the value for kurtosis in the posttest RT was highly positive (3.00), indicating a pointy and heavy-tailed distribution. Therefore, we removed all RTs that were three standard deviations higher or lower than the mean of the correct posttest RTs $(M=3481.75, S D=39.31)$ and pretest RTs $(M=4283.64, S D=$ 69.60). This resulted in skewness and kurtosis values of almost zero, indicating a normal distribution. After aggregating the data on the mean RT for every participant, we removed the participants with an accuracy below $60 \%$ to prevent that participants who were not mentally rotating and just guessing would blur potential small effects. First, we analyzed the data of condition 1 (S-3D with pretest and posttest) and condition 2 (2D with pretest and posttest). A mixed design ANOVA with test session (pre or post) as within variable and treatment (2D or S-3D) as between variable was executed. There was a main effect of test session, $F(1,15)=24.606, p<0.001, r=0.78$, which resembles the well-known training effect involved with the mental rotation paradigm (for a review, $\mathrm{se}^{26}$ ). The interaction between test session (pre or post) and treatment (2D or S-3D) was not significant, $F(1,15)<1, r=0.06$. The histogram shows that the means are highly similar, indicating that mental rotation performance after watching a movie in S-3D does not alter differently compared with after watching a movie in 2D (Fig. 2). The analyses described until now did not include the data of conditions 3 and 4 . Therefore, a one-way ANOVA with the posttest RTs as dependent variable (mean RT over rotation angles) and treatment (2D or S-3D) as the factor was executed. Again, we used an accuracy cut-off of $60 \%$. The effect of treatment was again not significant, $F(1,19)=3.78, p=0.07, r=0.41$.

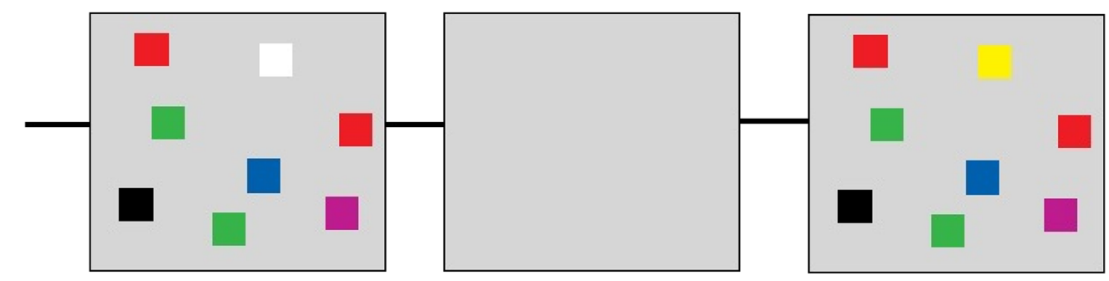

FIGURE 1 - An example of a trial in the change detection task. A sample array (left) is presented for $100 \mathrm{~ms}$, followed by a blank interval (middle) of $900 \mathrm{~ms}$. Next, the test array (right) appears and the participant has to indicate if something has changed or not. 


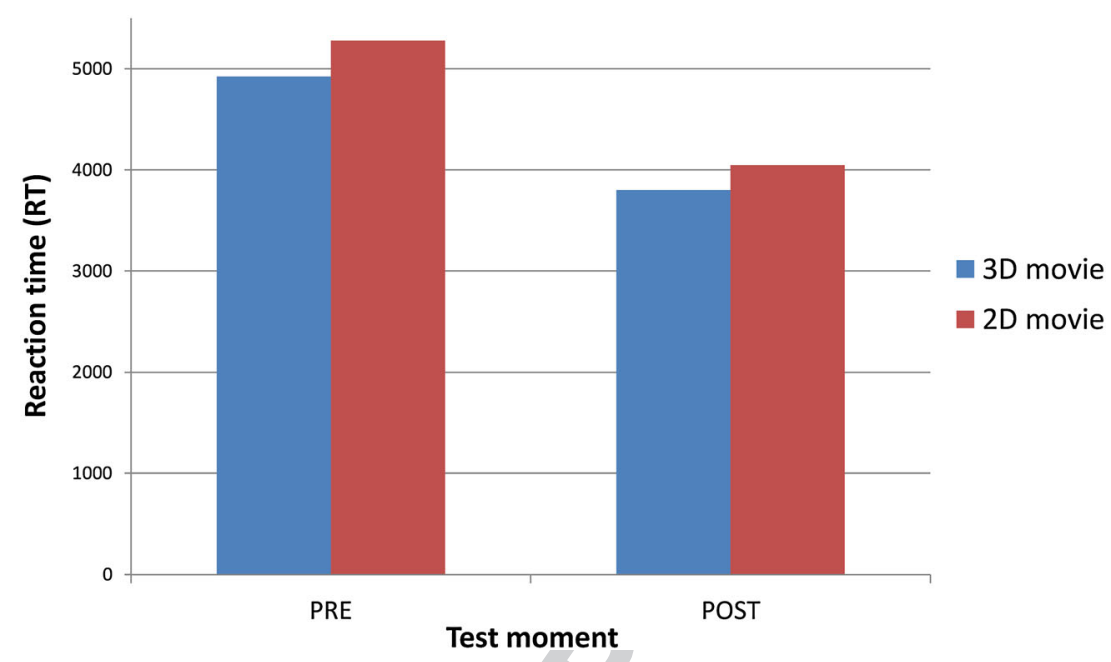

FIGURE 2 - The average reaction times (in $\mathrm{ms}$ ) in the mental rotation task, displayed for test moment (pre or post) and movie type (2D or S-3D).

\subsubsection{Distance estimation task}

In this analysis, the dependent variable was distance deviation (absolute value of actual distance - walked distance). A mixed design ANOVA with test session (pre or post) and distance (the six different distances) as within variables was executed. Similar to the previous analyses, treatment (S-3D or 2D) was the between variable. Mauchly's test indicated that the assumption of sphericity had been violated for the main effect of distance, $\chi^{2}(14)=82.29, \quad p<0.05$ and the interaction between test session and distance, $\chi^{2}(14)=54.35, p<0.05$. Therefore, degrees of freedom were corrected using Greenhouse-Geisser estimates of sphericity $(\varepsilon=0.42$ for the main effect of distance and $\varepsilon=0.42$ for the interaction). We found a main effect of distance, $F(2.12,59.42)=24.22$, $p<0.001, r=0.68$. With increasing distances, the difference between the actual distance and the walked distance is also significantly increasing. The main effect of test session was not significant, $F(1,28)=0.83, p>0.05, r=0.17$, and also the three-way interaction between test session, distance and treatment was not significant, $F(2.99,83.58)=1.35, p>0.05$, $r=0.21$. Furthermore, the analysis on the posttest data of conditions 3 and 4 failed to find significant effects of S-3D $(p>0.05)$.

\subsubsection{Change detection task}

The first 10 trials of every participant were again removed because they were considered as training. Afterwards, we removed all trials with an incorrect response. We again checked if our RTs were normally distributed. We saw that the value for kurtosis in the posttest RT was highly positive (1.72), indicating a pointy and heavy-tailed distribution. Therefore, we removed all RTs that were three SDs higher or lower than the mean of the correct posttest RTs $(M=1743.80, S D=267.17)$ and pretest RTs $(M=1783.78$, $S D=293.35)$. This resulted in skewness and kurtosis values of almost zero, indicating a normal distribution. The data was aggregated on the mean RT and accuracy per test session for every participant, and per number of colored squares on the screen. Trials with 8 or 12 squares on the screen were excluded from further analyses because they were too difficult and therefore of no use for our experiment. Next, we removed participants with an accuracy lower than $70 \%$ for 2,3 , and 4 squares and $55 \%$ for 6 squares. A mixed design ANOVA was executed with test session (pre or post) and number of on-screen squares $(2,3,4$, or 6$)$ as within variables and treatment as between variable. We found a significant main effect of test session, $F(1,6)=9.84, p<0.05, r=0.79$, and number of on-screen squares, $F(3,18)=13.06, p<0.001$, $r=0.83$. The three-way interaction between test session, number of colored squares and treatment was not significant, $F(3,18)=0.80, p>0.05, r=0.34$

The analysis was repeated with the accuracy as dependent variable, but no main and interaction effects were significant. Also the analysis on the posttest data of conditions 3 and 4 failed to find significant effects of S-3D $(p>0.05)$.

\subsubsection{Verbal fluency task}

The dependent variable in this task was the total number of words participants were able come up with in $60 \mathrm{~s}$. Per test session, their score was the sum of the performance for two different letters. There were four different letters, which were counterbalanced over participants. A mixed design ANOVA with test session (pre or post) as within variable and treatment as between variable revealed no significant results. The main effect of test session was not significant, $F(1,28)=2.62$, $p>0.05, r=0.29$, indicating that there is no training effect involved in verbal fluency (participants' performance was not increasing). Also the interaction between testing and treatment was not significant, $F(1,28)=0.28, p>0.05, r=0.1$. For conditions 3 and 4, we did a one-way ANOVA with total number of words in the posttest as dependent variable and treatment as between variable. The effect of treatment was not significant, $F(1,30)=0.42, p>0.05, r=0.12$. 


\subsubsection{Order effects}

Because the design of this experiment was rather complex (pretests and posttests, four different tasks), we looked at possible order effects (the order of the tasks was counterbalanced across participants). A factorial ANOVA with the posttest RT of the visual change detection task as dependent variable and treatment (3D or 2D) and order of tasks (MRT - Distance Visual, Distance -Visual -MRT, Visual - MRT - Distance) as factors was executed. We found a significant main effect of order, $F(2,31)=7.42, p<0.05, r=0.57$, but a nonsignificant interaction between order and treatment, $F(2,31)=2.19$, $p>0.05, r=0.35$. It is therefore very unlikely that task order played a role in our results.

\subsection{Discussion experiment 1}

We expected that visual fatigue effects on the perceptual level (as previously shown in e.g., ${ }^{8}$ ) would also affect cognitive processing performance on higher levels. In contrast to our hypothesis, the general tendency in the results of the different tasks is that S-3D has no observable effect on our cognitive system. First, there were no significant differences between the 2D and S-3D conditions in mental rotation performance. This lack of effect could not be attributed to a type II error (failing to observe a difference that actually exists), because our experiment did have enough power to detect the basic effects of the tasks. There was a significant training effect and a significant effect of mental rotation angle. The first replicates a stable finding in the mental rotation literature: performance improves with training. ${ }^{27}$ The second is the core finding of mental rotation: the bigger the rotation angle, the longer the mental rotation lasts. ${ }^{14}$ Neither reaction time nor accuracy yielded a clear difference between the conditions. Second, third, and fourth, the visual change detection task, ${ }^{20}$ the distance estimation task (real life spatial cognition) and the verbal fluency task (general cognitive fatigue) did not show any difference between 2D and S-3D conditions.

Thus we conclude that there are no observable aftereffects of watching a movie in S-3D on these cognitive processes.

\section{Experiment 2}

Much optometric research on the topic of visual discomfort related to S-3D viewing has shown an effect on low-level perceptual processes (e.g. $\left.{ }^{8,9}\right)$. Therefore, we wanted to focus more deeply on a cognitive process which takes place early in the stream of information processing, namely the visual attention. An important paradigm within the field of visual attention is the visual search paradigm. ${ }^{28}$ In a visual search task, participants typically have to indicate if a target object is present or not in a display with a number of distractor objects. Our research question was if visual fatigue would affect visual search performance. It has been shown that eye movement ability has a significant effect on visual search ${ }^{29}$ : if S-3D fatigues the eye muscles, it could be possible that participants will be slower and/or less accurate. A second experiment with a pretest-posttest design was set up to investigate this question.

\subsection{Method \\ 3.1.1 Participants}

A total of 32 students ranging from 18 to 26 years $(M=21.53$, $S D=2.37)$ participated in the experiment and were paid $€ 10$ for participation.

\subsubsection{Experimental set-up}

Both 2D and S-3D images were shown on a Philips 46" 9000 LED series television. Because we did not find any effect of movie content in the previous study, we now used two different movies, that is, "Step up 3" and "Alice in Wonderland". For each movie, we had again a 2D and a S-3D version. Instead of using a full-length movie, participants watched for $60 \mathrm{~m}$, which is the length mostly used in previous research., ${ }^{8,6}$

\subsubsection{Visual search task}

We programmed a visual search task comparable with that of Treisman and Gelade ${ }^{28}$ by using displays with $1,5,15,30$, and 50 stimuli. The distractors were brown letters "T" $(50 \%)$ or green letters "X" (50\%). The target was a blue letter or the letter "S". There were 160 randomized trials, including two replications of 80 different displays. A trial started with the presentation of a fixation cross for $1500 \mathrm{~ms}$. This fixation display was immediately followed by the stimulus display (see Fig. 3). Participants were instructed to answer as fast and correct as possible by pressing the right button when the target was present and pressing the left button when the target was absent. In case of an error, feedback was given ("incorrect!") to ensure that participants did not forget the instructions of the task and to encourage them to be more correct. The dependent variable of interest was the reaction time in milliseconds.

\subsubsection{Procedure}

At their arrival, participants filled in the standard informed consent for experiments at the faculty. All of the participants had to take part in a pretest and a posttest session. In both sessions they conducted the visual search task. The settings and the apparatus of the lab were the same as in experiment 1 . In the posttest, all participants filled in a questionnaire about their visual fatigue to check if there was a relationship between the individual perception of visual discomfort and the task performance (cf. Yang et $a l .{ }^{25}$ ) This questionnaire was based on the negative effects section of the ITC- Sense of Presence Inventory ${ }^{30}$ and consisted of 13 items measured on a 5-point Likert scale.

\subsection{Results}

\subsubsection{Visual discomfort}

Exploratory factor analysis was used to check if the 13 items in the visual discomfort questionnaire did measure the visual 

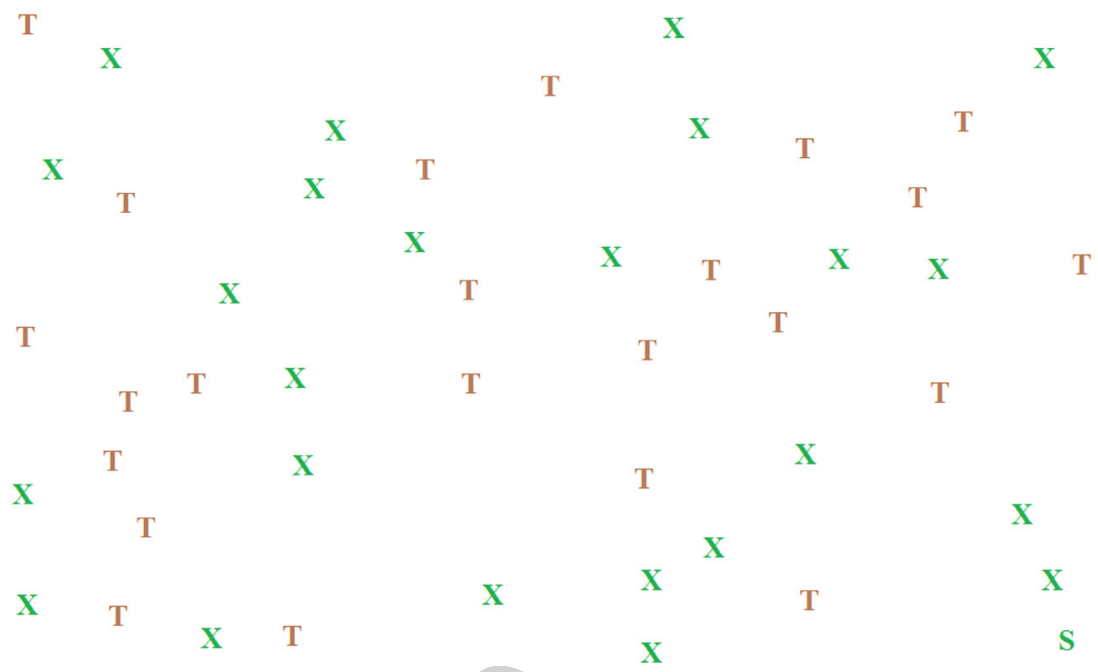

FIGURE 3 - An example of a trial in the visual search task. A number of stimuli are presented and the participant has to indicate if a target is present or not. In this case, the target is a green letter " $\mathrm{S}$ ".

discomfort construct. Two items were excluded because of poor factor loadings ("I had dry eyes" and "I had stiff shoulders and a stiff neck"). A two-way ANOVA with the self-reported visual discomfort as dependent variable and the variables endition (2D or S-3D) and movie (Alice in Wonderland or Step Up 3) as factors was executed. Results showed a main effect of endition, $F(1,28)=11.06, p=0.002, r=0.53$, indicating that people who saw the movie in S-3D experienced more visual discomfort. The effect of movie and the interaction between movie and eondition were not significant.

\subsubsection{Visual search task}

For every participant, the first 10 trials were excluded because they were considered as a training phase. Next, we removed all incorrect trials. We then checked if our RTs were normally distributed. We saw that the value for kurtosis was highly positive in both pretest RTs (2.72) and posttest RTs (2.71), indicating a pointy and heavy-tailed distribution. Therefore, all RTs 3SDs higher or lower than the mean of the correct pretest RTs $(M=1428.65, S D=806.40)$ and posttest RTs $(M=1301.97, S D=704.34)$ were removed. This resulted in skewness and kurtosis values of almost zero ( 0.72 for pretest RTs and 0.74 for posttest RTs), indicating a normal distribution. The data was aggregated on the mean RT per test session for every participant (pre or post), per number of stimuli on the screen $(1,5,15,30$, or 50 stimuli) and per presence of the target (yes or no).

First, we looked at the RT in which the target was present F4 (Fig. 4). A mixed design ANOVA with test session (pre or post) and display size $(1,5,15,30$ or 50 on-screen stimuli) as within variables, treatment $(2 \mathrm{D}$ or $\mathrm{S}-3 \mathrm{D})$ as between variable and visual discomfort as a covariate was executed. Mauchly's test indicated that the assumption of sphericity had been violated for the main effect of display size, $\chi^{2}(9)=31.88, p<0.05$.
Therefore degrees of freedom were corrected using Greenhouse-Geisser estimates of sphericity $(\varepsilon=0.68)$. There was a significant main effect of display size, $F(2.71,78.63)=6.06$, $p=0.001, r=0.41$, indicating that a large number of on-screen stimuli results in prolonged search duration. There was no significant effect of treatment (2D or S-3D), showing that the RTs of people in the 2D and S-3D condition were generally the same, $F(1,29)<1, r=0$. The effect of test session was not significant, $F(1,29)=1.27, F<1, r=0.20$, indicating that there was observable training effect from pretest to posttest. All other interactions were also not significant, $F<1$.

Second, we looked at the RT for trials in which the target was not present. A mixed design ANOVA with test session (pre or post) and display size $(1,5,15,30$, or 50 on-screen stimuli) as within variables, treatment (2D or S-3D) as between variable and visual discomfort as covariate was executed. Again, Mauchly's test indicated that the assumption of sphericity had been violated for the main effect of display size, $\chi^{2}(9)=123.12, p<0.05$. Therefore degrees of freedom were corrected using Greenhouse-Geisser estimates of sphericity $(\varepsilon=0.35)$. The effect of treatment $(2 \mathrm{D}$ or S-3D), was not significant, indicating that the RTs of people in the $2 \mathrm{D}$ and S-3D condition were generally the same, $F(1,29)<1, r=0.21$. Next to a significant main effect of display size, $F(1.41,40.84)=11.80$, $p<0.001, r=0.54$, there was also a significant main effect of test session (pre or post), $F(1,29)=5.77, p=0.02, r=0.41$. This last effect can be seen as a training effect.

\subsection{Discussion experiment 2}

Regarding the subjective measurements, there was a significant effect of condition (2D or S-3D) on self-reported visual discomfort: people who watched a movie in S-3D had more complaints about their viewing experience. The main research question in this second experiment was if S-3D would affect 

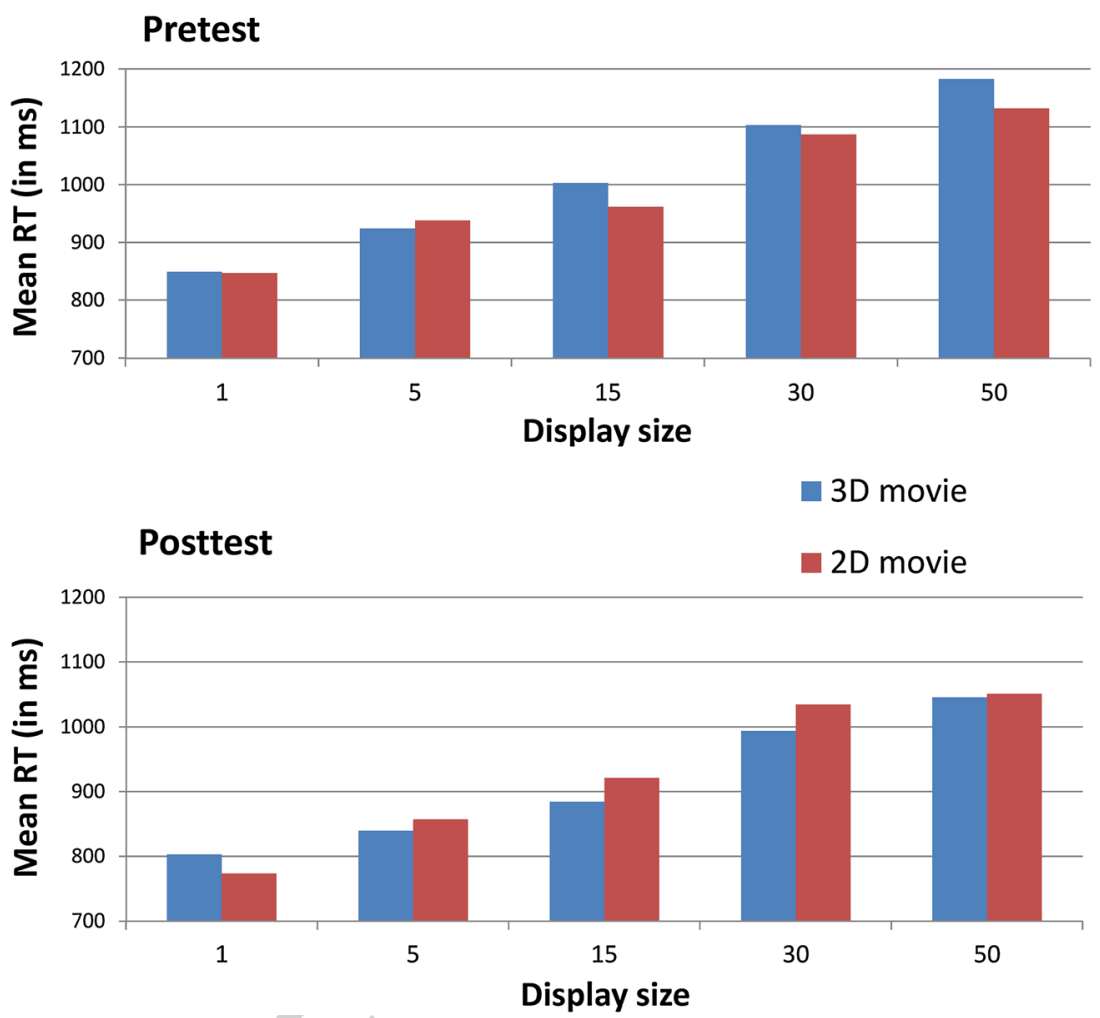

FIGURE 4 - The average reaction times (in ms) in the visual search task (target present), displayed for test moment (pre or post), display size $(1,5,15,30$, or 50), and movie type (2D or S-3D).

the performance in a classic visual search task. However, our findings show that there is no difference between the $2 \mathrm{D}$ and the S-3D condition in RT. For none of the display sizes, the mean RT differed significantly between the $2 \mathrm{D}$ and the S-3D group. As for experiment 1, we believe the task was sensitive enough to detect potential effects of S-3D and because we were able to replicate the basic effects of visual search, a type II error is unlikely.

\section{Conclusion}

Our motivation to study the cognitive aftereffects of S-3D was the observation that manufacturers use disclaimers advising consumers to watch S-3D only for a short period and to avoid car driving directly after exposure to stereoscopic viewing. Our aim was to test the scientific basis of these concerns by measuring the effect of S-3D on cognitive functioning by using widespread, validated techniques. Thereby, special attention was paid to the ecological validity of the design, so that results would be generalizable to real-life situations. At the moment, we are inclined to say there is no measurable effect of watching S-3D on cognitive functioning. We cannot firmly state that cognitive aftereffects of S-3D do not exist, but we can conclude that, if they do exist, they are apparently not measurable through measures of cognitive performance that have been widely used in different contexts for decades.
As a consequence, we believe that, even if there is an effect, its size is too small to affect more complex real-life tasks such as driving a car.

\section{References}

1 "User Manual Samsung UN55C7000 55" 1080p 3D LED HDTV." Samsung, Daegu, p. 19

2 M. R. Leary, "Introduction to Behavioral Research Methods: International Edition." Pearson Education, Limited (2011).

3 R. Patterson, "Human factors of 3-D displays," J. Soc. Inf. Display 15, 861-871 (2007).

4 E. Kronholm et al., "Self-reported sleep duration and cognitive functioning in the general population," J. Sleep Res. 18, No. 4, 436-46 (2009).

5 W. J. Tam et al., "Stereoscopic 3D-TV: Visual Comfort," IEEE Trans. Broadcast. 57, No. 99, 1-1 (2011).

6 S. Yano et al. " "A study of visual fatigue and visual comfort for 3D HDTV/ HDTV images," Displays 23, No. 4, 191-201 (2002).

$7 \mathrm{~S}$. Yano et al., "Two factors in visual fatigue caused by stereoscopic HDTV images," Displays 25, No. 4, 141-150 (2004).

$8 \mathrm{M}$. Emoto et al., "Changes in fusional vergence limit and its hysteresis after viewing stereoscopic TV," Displays 25(2-3), 67-76 (2004).

9 T. Inoue and H. Ohzu, "Accommodative responses to stereoscopic threedimensional display," Appl. Opt. 36, No. 19, 4509-15 (1997).

10 M. T. Banich, "Cognitive neuroscience and neuropsychology," Houghton Mifflin Co. (2004).

11 F. Biocca, "The cyborg's dilemma: embodiment in virtual environments," Proceedings, Second International Conference on Cognitive Technology: Humanizing the Information Age, 12-26 (1997).

12 K. Stanney and G. Salvendy, "Aftereffects and sense of presence in virtual environments: formulation of a research and development agenda," Int. J. Hum-Comput. Int. 10, No. 2, 135-187 (1998).

$13 \mathrm{~K}$. Behr et al., "Some practical considerations of ethical issues in VR research," Presence: Teleop. Virtual Environ. 14, No. 6, 668-677 (2005). 
14 R. N. Shepard and J. Metzler, "Mental rotation of three-dimensional objects," Science 171, No. 3972, 701 (1971).

15 I. Spence and J. Feng, "Video games and spatial cognition," Rev. Gen. Psychol. 14, No. 2, 92-104 (2010).

16 D. Stransky et al., "Mental rotation: cross-task training and generalization," J. Exp. Psychol. Appl. 16, No. 4, 349-60 (2010).

17 J. M. Loomis et al., "Visual space perception and visually directed action," J. Exp. Psychol. Hum. Percept. Perform. 18, No. 4, 906-21, (1992).

18 J. W. Philbeck, "Visually directed walking to briefly glimpsed targets is not biased toward fixation location," Perception 29, No. 3, 259-272 (2000).

19 B. J. Mohler et al., "The influence of feedback on egocentric distance judgments in real and virtual environments," in Proceedings of the 3rd Symposium on Applied Perception in Graphics and Visualization, 1, No. 212, (2006), pp. 9-14.

20 S. J. Luck and E. K. Vogel, "The capacity of visual working memory for features and conjunctions," Nature 390, No. 6657, 279-81 (1997).

21 R. L. Solomon, "An extension of control group design," Psychol. Bull. 46, 137-150 (1949)

22 M. Braver and S. L. Braver, "Statistical treatment of the Solomon four-group design: A meta-analytic approach," Psychol. Bull. 104, No. 1, 150 (1988).

23 S. G. Vandenberg and A. R. Kuse, "Mental rotations, a group test of threedimensional spatial visualization," Percept. Mot. Skills 599-604 (1978).

24 A. Basso and F. Burgio, "Semantic category and initial letter word fluency in left-brain-damaged patients," Eur. J. Neurol. No. 4, 544-550 (1997).

$25 \mathrm{~S}$. Yang et al., "Individual differences and seating position affect immersion and symptoms in stereoscopic 3D viewing," Optom. Vis. Sci. 1-44.

$26 \mathrm{M}$. Heil et al., "What is improved if a mental rotation task is repeated-the efficiency of memory access, or the speed of a transformation routine?" Psychol. Res. 61, No. 2, 99-106 (1998).

27 M. Peters et al., "A redrawn Vandenberg and Kuse mental rotations test: different versions and factors that affect performance," Brain Cogn. 28, No. 1, 39-58 (1995).

28 A. Treisman, "A feature-integration theory of attention," Cogn. Psychol. 136, 97-136 (1980).

29 L. K. Miller, "Developmental differences in the field of view during covert and overt search," Child Dev. 44, No. 2, 247-52 (1973).

$30 \mathrm{~J}$. Lessiter et al., "A cross-media presence questionnaire: the ITC-sense of presence inventory," Presence: Teleop. Virtual Environ. 10, No. 3, 282-297 (2001)

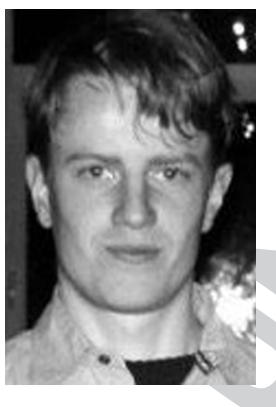

Klaas Bombeke obtained the degree of Master in the Experimental and Theoretical Psychology in June 2012 at Ghent University. Klaas was an intern at the research group for Media and ICT (iMindsMICT) at Ghent University, Belgium. Supervised by Professor Dr. Jan Van Looy, he conducted research into cognitive aftereffects of stereoscopic 3D movies. He works as a junior researcher at MICT, where he is involved in the 3DTV 2.0 project. Current topics include stereoscopic 3D user experience and aftereffects, and subjective video quality assessment methodology.

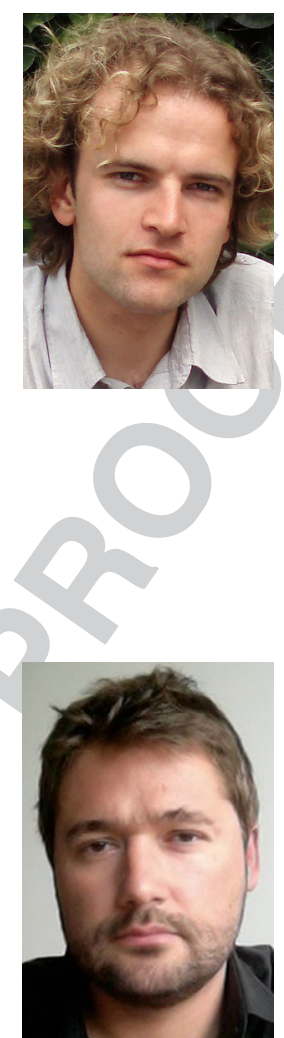

Jan Van Looy is assistant professor at the research group for Media and ICT (iMinds-MICT) at Ghent University, Belgium, where he and his team conduct multidisciplinary research into immersive media usage and gaming. Current topics include stereoscopic 3D user experience and aftereffects, omnidirectional video experience, subjective video quality assessment methodology, digital game usage, and experience and serious games effectiveness.

Arnaud Szmalec (born 1976) is an experimental psychologist who obtained a PhD in Psychological Sciences from Ghent University (2005). He is currently lecturer in Cognitive Psychology at the Université Catholique de Louvain (Belgium). He has a broad interest in cognitive science, studying both theoretical and applied research question in the domains of attention, memory, and language.

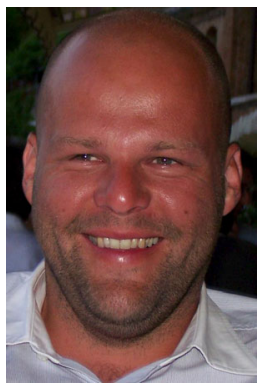

Wouter Duyck is an associate professor at the Department of Experimental Psychology, Ghent, Belgium. For his dissertation, he received the triennial Prix de Psychologie of the Royal Academy of Science, Humanities and Fine Arts of Belgium, awarded to the best doctoral dissertation in psychology. He has authored or co-authored several book chapters and about 40 scientific articles, most of which in the field of cognitive psychology. His main research interests include reading and the visual system, language acquisition, and working memory. 


\section{Author Query Form}

\section{Journal: Journal of the Society for Information Display}

\section{Article: jsid_164}

Dear Author,

During the copyediting of your paper, the following queries arose. Please respond to these by annotating your proofs with the necessary changes/additions.

- If you intend to annotate your proof electronically, please refer to the E-annotation guidelines.

- If you intend to annotate your proof by means of hard-copy mark-up, please refer to the proof mark-up symbols guidelines. If manually writing corrections on your proof and returning it by fax, do not write too close to the edge of the paper. Please remember that illegible mark-ups may delay publication.

Whether you opt for hard-copy or electronic annotation of your proofs, we recommend that you provide additional clarification of answers to queries by entering your answers on the query sheet, in addition to the text mark-up.

\begin{tabular}{|c|l|c|}
\hline Query No. & \multicolumn{1}{|c|}{ Query } \\
\hline Q1 & $\begin{array}{l}\text { AUTHOR: As per journal style, please confirm SID membership status of each author } \\
\text { (Student Member, Member, Senior Member, Fellow, Life Member, Life Senior Member, } \\
\text { Life Fellow) }\end{array}$ \\
\hline Q2 & AUTHOR: Please provide the city location of publisher for Reference 2. \\
\hline Q3 & AUTHOR: Please provide the city location of publisher for Reference 10. \\
\hline Q4 & AUTHOR: Please provide volume number for reference 23. \\
\hline Q5 & AUTHOR: Please provide volume number for reference 24. \\
\hline Q6 & AUTHOR: Please provide volume number and date for reference 25. \\
\hline
\end{tabular}


Required software to e-Annotate PDFs: Adobe Acrobat Professional or Adobe Reader (version 7.0 or above). (Note that this document uses screenshots from Adobe Reader $\mathrm{X}$ )

The latest version of Acrobat Reader can be downloaded for free at: http://get.adobe.com/uk/reader/

Once you have Acrobat Reader open on your computer, click on the Comment tab at the right of the toolbar:

닙

This will open up a panel down the right side of the document. The majority of tools you will use for annotating your proof will be in the Annotations section, pictured opposite. We've picked out some of these tools below:

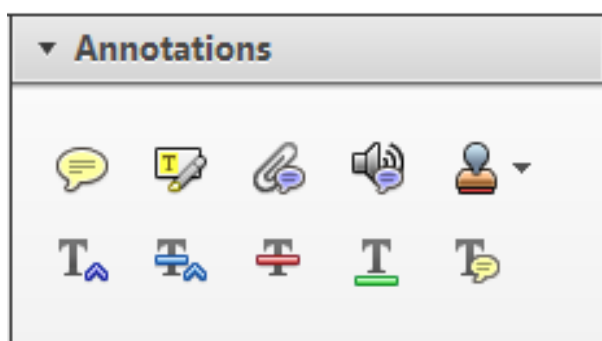

1. Replace (Ins) Tool - for replacing text.

Strikes a line through text and opens up a text box where replacement text can be entered.

How to use it

- Highlight a word or sentence.

- Click on the Replace (Ins) icon in the Annotations section.

- Type the replacement text into the blue box that appears.

Idard tramework for the analysis of $\mathrm{m}$ icy-Nevertheless, it also led to exog،

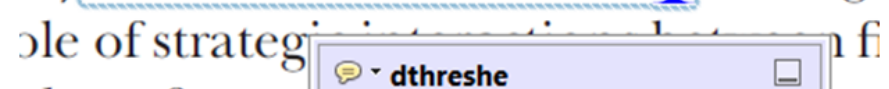
\begin{tabular}{l|l|l} 
aber of comp & 08/06/2011 15:58:17 & 0
\end{tabular} is that the $\mathrm{s} 1 \overline{\text {, which led }}$ of nain compo: be level, are exc nc

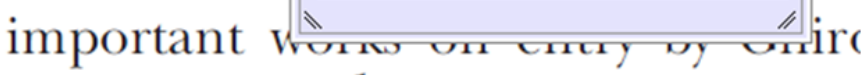
M heneferth) ${ }^{1}$ we anen the "hlarl $\mathrm{h}$

3. Add note to text Tool - for highlighting a section to be changed to bold or italic.

T Highlights text in yellow and opens up a text box where comments can be entered.

How to use it

- Highlight the relevant section of text.

- Click on the Add note to text icon in the Annotations section.

- Type instruction on what should be changed regarding the text into the yellow box that appears.

namic responses of mark ups ent with the VAR evidence

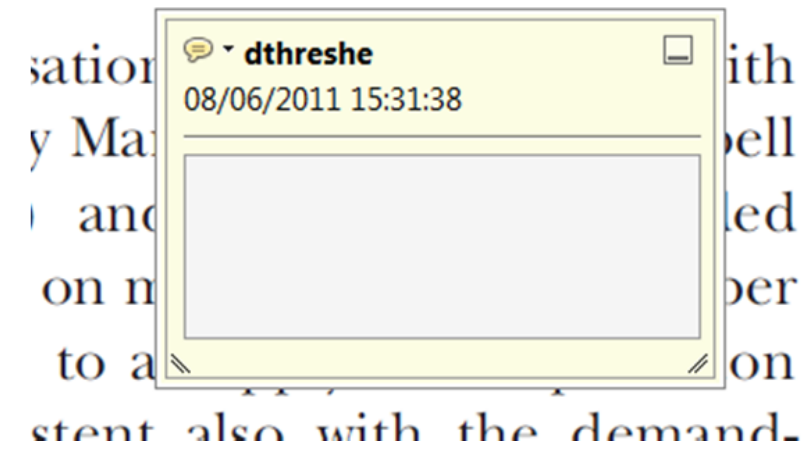

2. Strikethrough (Del) Tool - for deleting text.

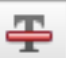

Strikes a red line through text that is to be deleted.

How to use it

- Highlight a word or sentence.

- Click on the Strikethrough (Del) icon in the Annotations section.

there is no room tor extra prohts al s ups are zero and the number of ret) values are not determined by Blanchard and Kiyotaki (1987), sfect competition in general equilil ts of aggregate demand and supply lassical framework assuming monol sen an evorenous number of firms

4. Add sticky note Tool - for making notes at specific points in the text.

Marks a point in the proof where a comment needs to be highlighted.

How to use it

- Click on the Add sticky note icon in the Annotations section.

- Click at the point in the proof where the comment should be inserted.

- Type the comment into the yellow box that appears.

iaisu airu suppiy sisuks. hivsl ui

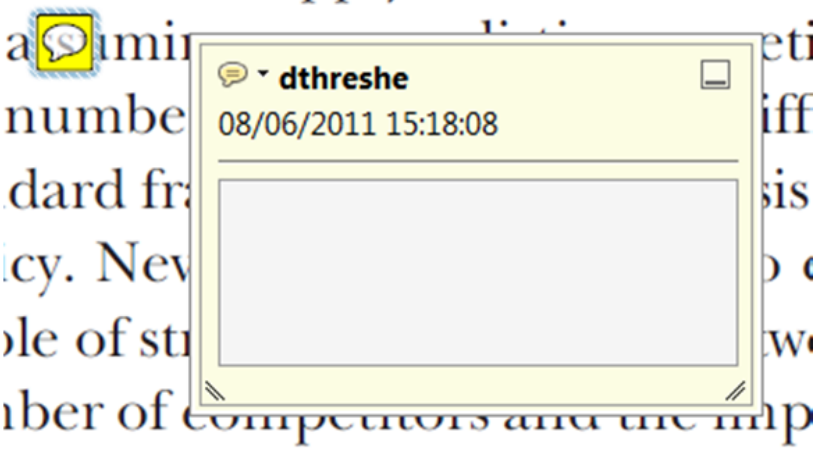

is that the structure of the sectc. 
5. Attach File Tool - for inserting large amounts of text or replacement figures.

Inserts an icon linking to the attached file in the appropriate pace in the text.

How to use it

- $\quad$ Click on the Attach File icon in the Annotations section.

- Click on the proof to where you'd like the attached file to be linked.

- Select the file to be attached from your computer or network.

- Select the colour and type of icon that will appear in the proof. Click OK.

E N D

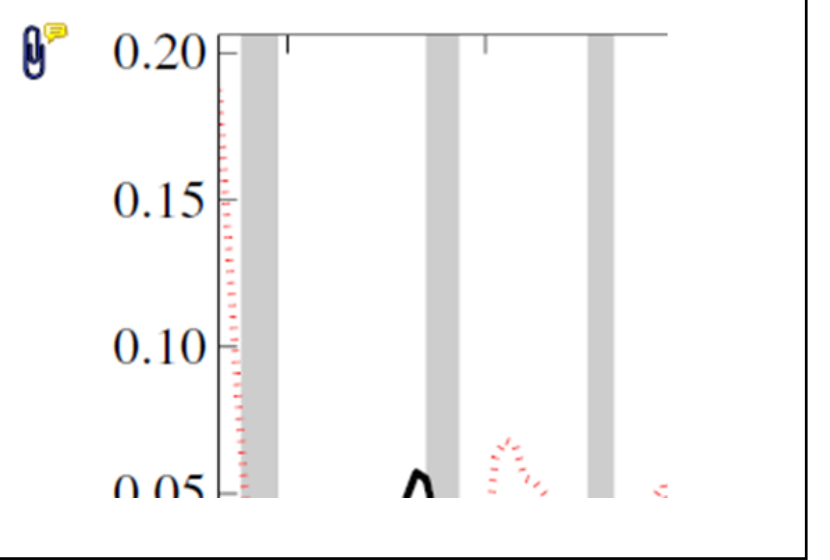

6. Add stamp Tool - for approving a proof if no corrections are required.

- Inserts a selected stamp onto an appropriate place in the proof.

\section{How to use it}

- $\quad$ Click on the Add stamp icon in the Annotations section.

- Select the stamp you want to use. (The Approved stamp is usually available directly in the menu that appears).

- Click on the proof where you'd like the stamp to appear. (Where a proof is to be approved as it is, this would normally be on the first page).

)t the Dusiness cycie, starting with the on perfect competition, constant ret

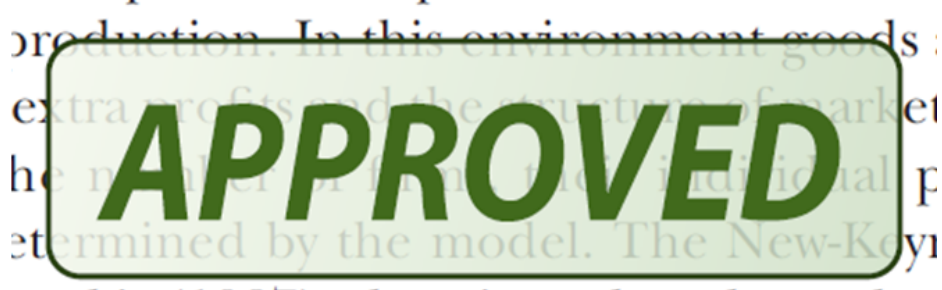
otaki (1987), has introduced produc general equilibrium models with nomin:

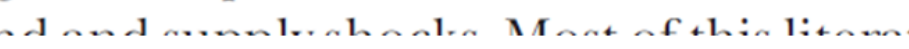

- Drawing Markups

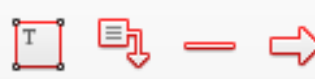

$0 \bigcirc \sqrt{6} \otimes$

\section{How to use it}

- Click on one of the shapes in the Drawing Markups section.

- Click on the proof at the relevant point and draw the selected shape with the cursor.

- To add a comment to the drawn shape, move the cursor over the shape until an arrowhead appears.

- Double click on the shape and type any text in the red box that appears.
7. Drawing Markups Tools - for drawing shapes, lines and freeform annotations on proofs and commenting on these marks.

Allows shapes, lines and freeform annotations to be drawn on proofs and for comment to be made on these marks.

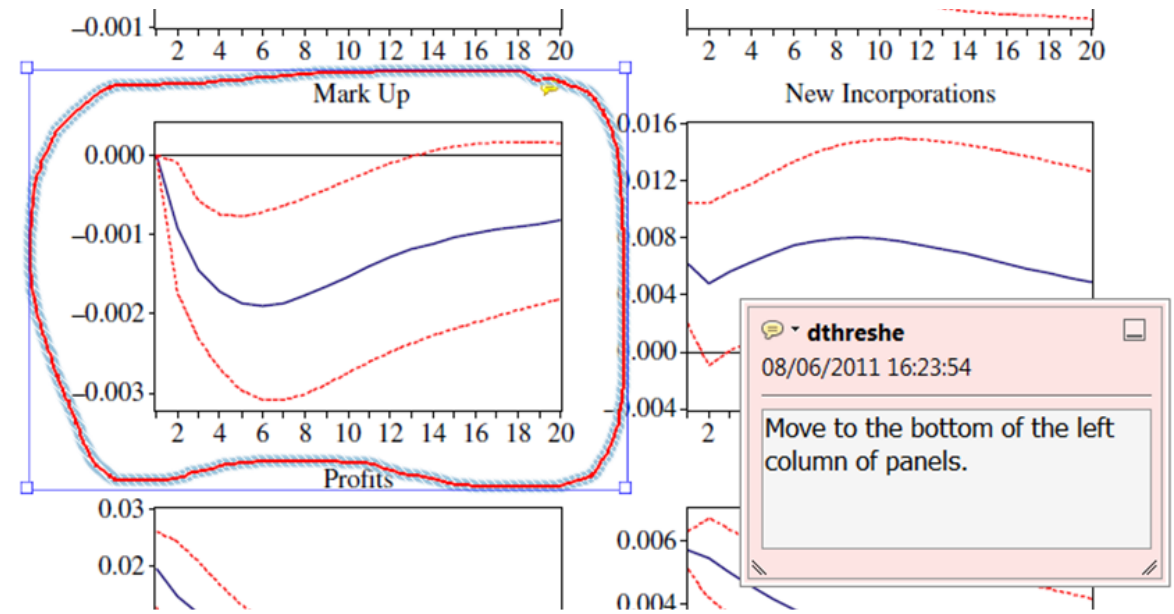

For further information on how to annotate proofs, click on the Help menu to reveal a list of further options:

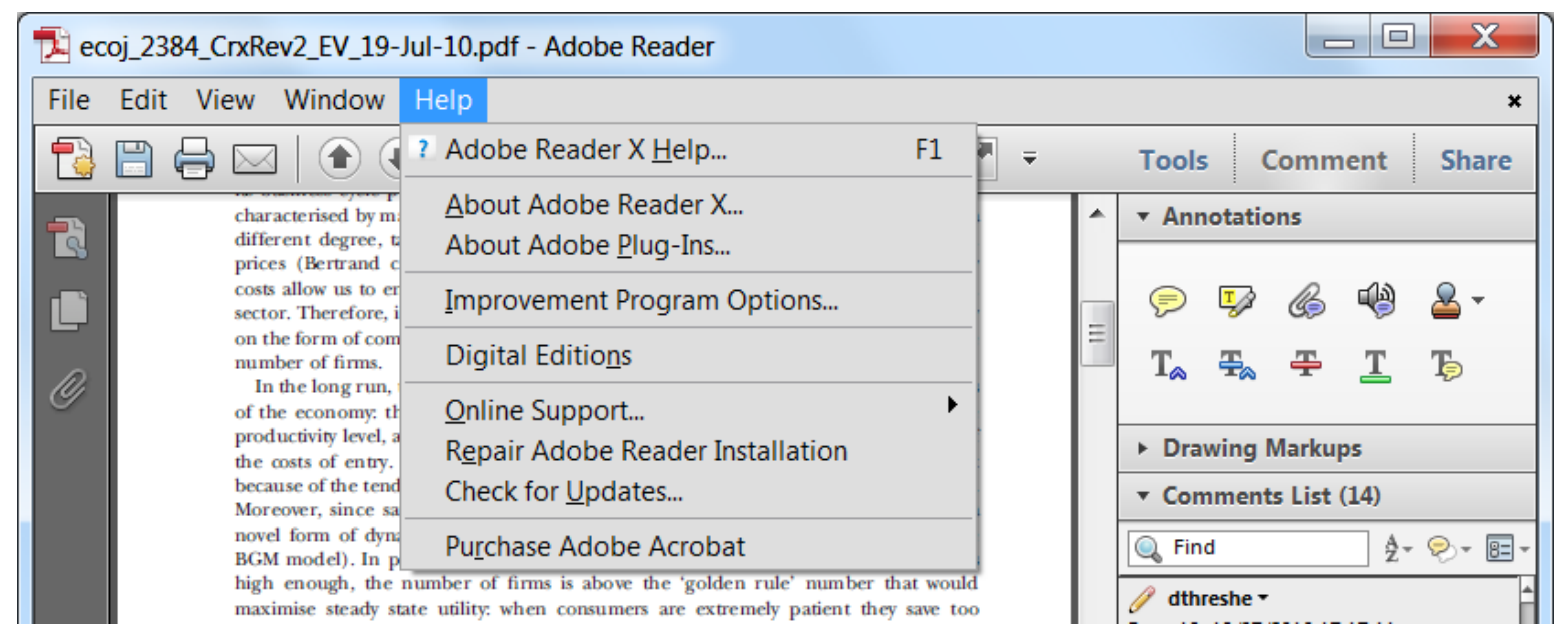

